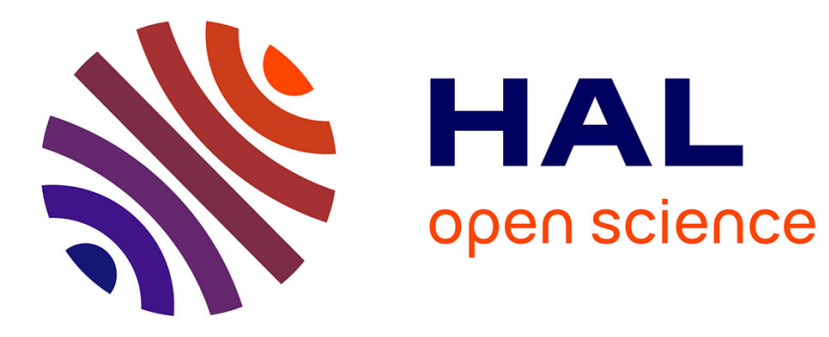

\title{
Non-Invasive Green Small Cell Network
}

Baher Mawlawi, Ejder Bastug, Chahé Nerguizian, Sylvain Azarian, Mérouane Debbah

\section{- To cite this version:}

Baher Mawlawi, Ejder Bastug, Chahé Nerguizian, Sylvain Azarian, Mérouane Debbah. Non-Invasive Green Small Cell Network. Asilomar 2012, Nov 2011, Pacific Grove, United States. 6p. hal-00771216

\section{HAL Id: hal-00771216 \\ https://hal-centralesupelec.archives-ouvertes.fr/hal-00771216}

Submitted on 8 Jan 2013

HAL is a multi-disciplinary open access archive for the deposit and dissemination of scientific research documents, whether they are published or not. The documents may come from teaching and research institutions in France or abroad, or from public or private research centers.
L'archive ouverte pluridisciplinaire HAL, est destinée au dépôt et à la diffusion de documents scientifiques de niveau recherche, publiés ou non, émanant des établissements d'enseignement et de recherche français ou étrangers, des laboratoires publics ou privés. 


\title{
Non-Invasive Green Small Cell Network
}

\author{
Baher Mawlawi $^{\star, \diamond}$, Ejder Baştuğ $\breve{g}^{\star}$, Chahé Nerguizian ${ }^{\dagger}$, Sylvain Azarian`, Mérouane Debbah ${ }^{\star}$ \\ $\star$ Alcatel-Lucent Chair - SUPÉLEC, Gif-sur-Yvette, France \\ $\diamond$ Department of Telecommunications Engineering, SUPÉLEC, Gif-sur-Yvette, France \\ $\dagger$ École Polytechnique de Montréal, Canada
}

\begin{abstract}
Future low cost wireless networks are expected to provide high data rates with low power consumption. A dense deployment of distributed small-cells, within the existing network infrastructure, is one of the candidate solutions to achieve this goal. Unfortunately, the aggregate signal resulting from the transmission of these multiples small cells can be considered as an electromagnetic (EM) pollution for passive users who do not carry wireless devices. These users are victim of primary electromagnetic "smokers" and request from operators to be spared from these new base stations. The aim of this contribution is to propose an electromagnetic friendly environment with minimum EM pollution, while satisfying the quality of service requirements. The technique employed, called Distributed SpaceTime Reversal (DSTR), focuses the energy on active users (equipped with wireless devices) and is able to spare passive users from EM waves. In this contribution, we provide a theoretical analysis of the technique and show the impact of active/passive users with respect to the number of cells.
\end{abstract}

\section{INTRODUCTION}

Recently, wireless networks are experiencing an evergrowing mobile devices' traffic demand [1]. This is causing a non-negligible increase of the electromagnetic (EM) pollution in the environment. Therefore, the concept of "Green Communications" [1] has recently received considerable attention. It consists of finding intelligent and innovative solutions to improve the energy efficiency, to reduce the electromagnetic pollution on passive users and to enhance the performance metrics of wireless communications in dense networks where more and more devices have to transmit.

Few methods can be used to minimize the radio waves impinging passive users. One of the considered strategies supports algorithms performing real time users' localization [2]-[4] and differentiation between people with and without wireless devices. Then, beamforming and beamnulling are applied for active and passive users, respectively. In this approach, the angular resolution of the antennas, as well as the real time user's localization, are considered challenging issues. A possible alternative is the adoption of time reversal techniques [5]. The operation principle of the TR technique consists in exploiting the environmental multi-path and the channel reciprocity, to focus temporally and spatially an electromagnetic (EM) signal towards the active users [6], [7].

Time reversal (TR) is used in acoustics and ultrasound domains [8], [9] since more than a decade, while the application of TR method in wireless communications is quite recent [5], [10]-[12]. In [5], a TR approach is used to alleviate the interference that a transmitter may cause to an un-intended receiver belonging to the same single cell with one antenna

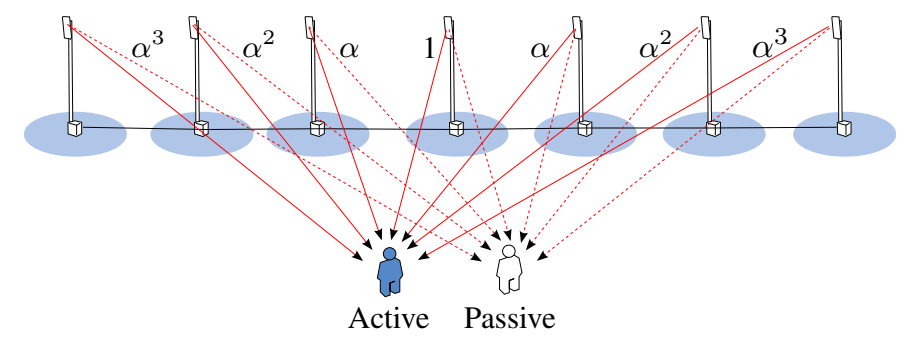

Figure 1. Illustration of active/passive users in a cooperative small cell network.

at the base station. The performance depends mainly on the propagation characteristics (number of paths, path loss as well as the power delay profile (PDP)).

In this contribution, we extend the analysis proposed in [5] to the case of a distributed multiple input multiple output (MIMO) small cell setting by replacing the delay domain by the space domain. We show in particular how the TR can be implemented in a distributed manner with multiple small cells even in the case of channels with a limited number of paths. The performance is analyzed theoretically and assessed by simulations to show the reduction in terms of EM pollution towards passive users.

The rest of the paper is organized as follows. Section II introduces the multipath channel and the system model. Section III gives the performance of a TR-based transmission in terms of pollution on passive users. Section IV shows how the active user's location affects the overall system performance. Simulation results and analysis are presented in Section V whereas conclusions are drawn in Section VI.

\section{SySTEM MODEL}

\section{A. Channel Model}

We consider a single input single output channel where the received signal can be written as [13]

$$
y(t)=\sum_{l=0}^{L-1} c_{l}(t) x\left(t-\tau_{l}\right)
$$

This may be interpreted as the output of a complex baseband time-varying linear channel with an impulse response equal to

$$
c(t, \tau)=\sum_{l=0}^{L-1} c_{l}(t) \delta\left(\tau-\tau_{l}(t)\right) .
$$


After sampling, the channel can be expressed as:

$$
h(k, t)=\sum_{l=0}^{L-1} c_{l}(t) g\left(k T-\tau_{l}\right),
$$

where $T$ is the sampling period and $\mathrm{g}$ is the filter at the transmitter side. Note that, in this paper, we assume slow fading and time invariant channels for both active and passive users. The discrete representation of the channel impulse response (CIR) at time $k$ may be modeled as

$$
\begin{aligned}
& h_{a}[k]=\sum_{l=0}^{L_{a}-1} h_{a, l} \delta[k-l], \\
& h_{p}[k]=\sum_{l=0}^{L_{p}-1} h_{p, l} \delta[k-l],
\end{aligned}
$$

where $h_{a, l}=h_{a}[l], L_{a}$ and $h_{p, l}=h_{p}[l], L_{p}$ represent the $l^{\text {th }}$ tap complex amplitude and the number of taps of the channel between the transmitter and an active and passive user, respectively. To gain some insight into the TR system while keeping the model analytically tractable, the CIRs associated with different receivers at different locations are assumed to be independent, i.e., the paths of each CIR are uncorrelated. Moreover, each $h_{a}[l], h_{p}[l]$ is a random variable with associated means $\left(m_{a}, m_{p}\right)$ and energy.

$$
\begin{aligned}
& E\left[\left|h_{a}[l]\right|^{2}\right]=P^{a}[l] \\
& E\left[\left|h_{p}[l]\right|^{2}\right]=P^{p}[l],
\end{aligned}
$$

where $P D P^{a}=\left[P^{a}[0], P^{a}[1], \ldots P^{a}[L-1]\right], P D P^{p}=$ $\left[P^{p}[0], P^{p}[1], \ldots P^{p}[L-1]\right]$ are the vectors of power delay profile for active and passive users respectively.

\section{B. TR Description}

In traditional TR-systems, the end user transmits a delta-like pilot pulse which propagates to the base station through the multi-path channel. Subsequently, the base station estimates the channel and time reverses the received waveform. A normalized time reversed version of the received signal is then used as a basic waveform, obtained as

$$
g[k]=\frac{h_{a}^{*}\left[L_{a}-1-k\right]}{\sqrt{E\left[\sum_{l=0}^{L_{a}-1}\left|h_{a}[l]\right|^{2}\right]}}, k=0,1, \ldots, L_{a}-1 .
$$

The multi-path channel forms a natural matched filter to the basic waveform $g[k], k=0,1, \ldots, L_{a}-1$, and hence a peak is expected at the receiver [5].

The base station loads the data stream on the basic waveform and transmits the signal to the end user.. In practice, since the baud rate is much lower than the sampling rate, an up/down sampling (Fig. 2) is performed using a rate back-off factor $D$ equal to the ratio of the sampling and the baud rate [14].

Let us consider the simple case given by a single antenna base station, an active and a passive user. If the sequence of information symbols are denoted by $x[k]$ and assumed to be

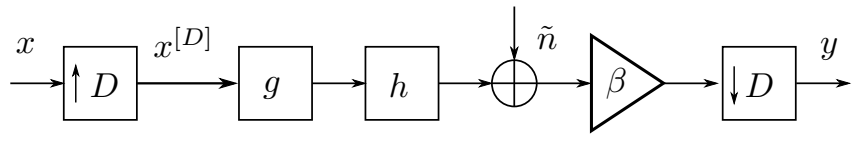

Figure 2. The block diagram of a TR-based communication system.

i.i.d complex random variables with zero mean and a variance $P$, the transmitted signal can be expressed as:

$$
s[k]=\left(x^{[D]} * g\right)[k]
$$

where $x^{[D]}[k]$ is an up-sampled sequence of $x[k]$ given by:

$$
x^{[D]}[k]= \begin{cases}x[k / D], & \text { if } k \bmod \mathrm{D}=0 \\ 0, & \text { if } k \bmod \mathrm{D} \neq 0\end{cases}
$$

The received signal $y[k]$ at the receiver is the result of the convolution of the transmitted signal $s[k]$ and $h[k]$ plus an additive white Gaussian noise $(A W G N) n[k]$ with zero mean and variance $\sigma^{2}$. The receiver simply performs a onetap gain adjustment to the received signal, i.e., a multiplication by a coefficient $\beta$, and then down-samples it with the back-off factor $D$. The signal before down-sampling can be written as:

$$
y^{[D]}[k]=\beta\left(x^{[D]} * g * h\right)[k]+\beta \tilde{n}[k] .
$$

Then, the down-sampled signal $y[k]$ is

$$
y[k]=\sum_{l=0}^{(2 L-2) / D} \beta(h * g)[D l] x[k-l]+\beta n[k],
$$

where $L$ is equal to the number of channel taps; with $k=0,1, \ldots, 2 L-2$, and $n[k]=\tilde{n}[D k]$, white Gaussian additive noise with zero mean and a variance of $\sigma^{2}$.

\section{PERformance AnAlysis}

In this section, we consider $M$ small-cells and compare the performance of the TR approach for active and passive users. Accordingly, we compute the signal to noise ratio (SNR) experienced by each user, and propose a strategy to minimize the EM pollution on the passive ones. Subscripts 'a' and 'p' are added to equation (11) to consider active and passive users, respectively.

\section{A. Active user's case}

The active user's received signal can be written as:

$$
y_{a}[k]=\sum_{i=1}^{M} \sum_{l=0}^{\left(2 L_{a i}-2\right) / D}\left(h_{a i} * g_{i}\right)[D l] x[k-l]+n_{a}[k] .
$$

By denoting $P$ as the power of the transmitted signal $x[k]$ and neglecting the noise term, the received power $P_{r}$ may be written as:

$$
\begin{aligned}
P_{r} & =E_{x}\left[\left|\sum_{i=1}^{M} \sum_{l=0}^{\left(2 L_{a i}-2\right) / D}\left(h_{a i} * g_{i}\right)[D l] x[k-l]\right|^{2}\right] \\
& =P\left|\sum_{i=1}^{M} \sum_{l=0}^{\left(2 L_{a i}-2\right) / D}\left(h_{a i} * g_{i}\right)[D l]\right|^{2},
\end{aligned}
$$




$$
\begin{aligned}
& A_{i}=\sum_{\substack{i=0 \\
i \neq\left(L_{a i}-1\right) / D}}^{\left(2 L_{a i}-2\right) / D} \sum_{\substack{j=0 \\
j \neq i \\
j \neq\left(L_{a i}-1\right) / D}}^{\left(2 L_{a i}-2\right) / D} \sum_{l=\max \left(i D-L_{a i}+1, j D-L_{a i}+1,0\right)}^{\min \left(L_{a i}-1, i D, j D\right)} P_{i}^{a}[l] m_{a i}^{*}\left[L_{a i}-1-i D+l\right] m_{a i}\left[L_{a i}-1-j D+l\right] \\
& +\sum_{\substack{i=0 \\
i \neq\left(L_{a i}-1\right) / D}}^{\left(2 L_{a i}-2\right) / D} \sum_{l=\max \left(i D-L_{a i}+1,0\right)}^{\min \left(L_{a i}-1, i D\right)} 2 m_{a i}[l]\left(P_{i}^{a}[l]-\frac{R e\left(m_{a i}[l]\right)^{2}}{2}\right) m_{a i}^{*}\left[L_{a i}-1-i D+l\right] \\
& +\sum_{\substack{j=0 \\
j \neq\left(L_{a i}-1\right) / D}}^{\left(2 L_{a i}-2\right) / D} \sum_{l=\max \left(j D-L_{a i}+1,0\right)}^{\min \left(L_{a i}-1, j D\right)} 2 m_{a i}^{*}[l]\left(P_{i}^{a}[l]-\frac{R e\left(m_{a i}[l]\right)^{2}}{2}\right) m_{a i}\left[L_{a i}-1-j D+l\right] \\
& B_{i}=\sum_{\substack{i=0 \\
i \neq\left(L_{a i}-1\right) / D}}^{\left(2 L_{a i}-2\right) / D} \sum_{\substack{j=0 \\
j \neq i \\
j \neq\left(L_{a i}-1\right) / D}}^{\left(2 L_{a i}-2\right) / D} \sum_{l=\max \left(i D-L_{a i}+1,0\right)}^{\min \left(L_{a i}-1, i D\right)} \sum_{\substack{k=\max \left(j D-L_{a i}+1,0\right) \\
k \neq l}}^{\min \left(L_{a i}-1, j D\right)} m_{a i}[l] m_{a i}^{*}[k] m_{a i}\left[L_{a i}-1-j D+k\right] m_{a i}^{*}\left[L_{a i}-1-i D+l\right] \\
& +\sum_{\substack{i=0 \\
i \neq\left(L_{a i}-1\right) / D}}^{\left(2 L_{a i}-2\right) / D} \sum_{l=\max \left(i D-L_{a i}+1,0\right)}^{\min \left(L_{a i}-1, i D\right)} \sum_{\substack{k=0 \\
k \neq l}}^{L_{a i}-1} P_{i}^{a}[k] m_{a i}[l] m_{a i}^{*}\left[L_{a i}-1-i D+l\right] \\
& +\sum_{\substack{j=0 \\
j \neq\left(L_{a i}-1\right) / D}}^{\left(2 L_{a i}-2\right) / D} \sum_{l=0}^{L_{a i}-1} \sum_{\substack{j D-L a i+1,0) \\
k \neq l}}^{\min \left(L_{a i}-1, j D\right)} P_{i}^{a}[l] m_{a i}^{*}[k] m_{a i}\left[L_{a i}-1-j D+k\right] \\
& C_{i}=\sum_{l=\max \left(D u-L_{a i}+1,0\right)}^{\min \left(L_{a i}-1, D u\right)} P_{i}^{a}[l] P_{i}^{a}\left[L_{a i}-1-D u+l\right] \\
& D_{i}=\sum_{l=\max \left(D u-L_{a i}+1,0\right)}^{\min \left(L_{a i}-1, D u\right)} \sum_{\substack{k=\max \left(D u-L_{a i}+1,0\right) \\
k \neq l}}^{\min \left(L_{a i}-1, D u\right)} m_{a i}[l] m_{a i}^{*}[k] m_{a i}\left[L_{a i}-1-D u+k\right] m_{a i}^{*}\left[L_{a i}-1-D u+l\right] \\
& E_{i}=\sum_{l=0}^{L_{a i}-1}\left[0.5\left(P_{i}^{a}[l]-\frac{\left(\operatorname{Re}\left(m_{a i}[l]\right)\right)^{2}}{2}\right)^{2}+\left(2 \operatorname{Re}\left(m_{a i}[l]\right)\right)^{2}\left(P_{i}^{a}[l]-\frac{\left(\operatorname{Re}\left(m_{a i}[l]\right)\right)^{2}}{2}\right)+4\left(\operatorname{Re}\left(m_{a i}[l]\right)\right)^{4}\right] \\
& F_{i}=\sum_{l=0}^{L_{a i}-1} \sum_{\substack{k=0 \\
k \neq l}}^{L_{a i}-1} P_{i}^{a}[l] P_{i}^{a}[k]
\end{aligned}
$$

where $E_{x}$ represents the expectation over $x$.

Since the signal-to-noise ratio (SNR) for the active user depends on each CIR realization, the average SNR is then given by

$$
E\left[S N R_{M I S O}^{a}\right]=\frac{E\left[P_{r}\right]}{\sigma_{a}^{2}} .
$$

Within this setting we have two cases:

$$
\begin{aligned}
& \begin{aligned}
& * \text { For }\left(L_{a i}-1\right) / D \in N \\
& E\left(S N R_{i}^{a}\right)=\frac{P}{\gamma \sigma_{a}^{2}}\left(A_{i}+B_{i}+\sum_{\substack{u=0 \\
u \neq\left(L_{a i}-1\right) / D}}^{\left(2 L_{a i}-2\right) / D}\left(C_{i}(u)+D_{i}(u)\right)\right. \\
&\left.+E_{i}+F_{i}\right)
\end{aligned}
\end{aligned}
$$

with $\gamma=\sum_{l=0}^{L_{a i}-1} P_{i}^{a}[l], A_{i}, B_{i}, C_{i}, D_{i}, E_{i}$ and $F_{i}$ given in equations (12), (13), (14), (15), (16) and (17) respectively.

$$
\text { * For }\left(L_{a i}-1\right) / D \notin N
$$

Due to lack of space, we do not provide the case for $\left(L_{a i}-1\right) / D \notin N$. It can be found in [15].

As $D$ increases (sampling rate much greater than the baud rate), the summation will reduce to only one term (first channel sample element) and the $E\left(S N R_{i}^{a}\right)$ will gradually decrease in both cases. Then,

$$
E\left(S N R_{M I S O}^{a}\right)=\sum_{i=1}^{M} E\left(S N R_{i}^{a}\right)+\frac{P}{\sigma_{a}^{2}} \sum_{i=1}^{M} \sum_{\substack{j=1 \\ j \neq i}}^{M} B_{i j}
$$

with $L_{a i}$ and $L_{a j}$ are equals to the number of channel taps between the active user and the considered small cell $i$ and $j$ respectively. $B_{i j}$ is given in equation (22). 


$$
\begin{aligned}
& \text { For }\left(L_{a i}-1\right) / D \in N,\left(L_{a j}-1\right) / D \in N \\
& B_{i j}=\sum_{\substack{u=0 \\
u \neq\left(L_{a i}-1\right) / D}}^{\left(2 L_{a i}-2\right) / D} \sum_{\substack{k=0 \\
k \neq\left(L_{a j}-1\right) / D}}^{\left(2 L_{a j}-2\right) / D} \frac{\sum_{l=\max \left(D u-L_{a i}+1,0\right)}^{\min \left(L_{a i}-1, D u\right)} m_{a i}[l] m_{a i}^{*}\left[L_{a i}-1-D u+l\right]}{\sqrt{\sum_{l=0}^{L_{a i}-1} P_{i}^{a}[l]}} \frac{\sum_{l=\max \left(D k-L_{a j}+1,0\right)}^{\min \left(L_{a j}-1, D k\right)} m_{a j}^{*}[l] m_{a j}\left[L_{a j}-1-D u+l\right]}{\sqrt[\sum_{l=0}^{L_{a j}-1} P_{j}^{a}[l]]{\sum_{l=0}}} \\
& +\sum_{\substack{u=0 \\
u \neq\left(L_{a i}-1\right) / D}} \frac{\sum_{l=\max \left(D u-L_{a i}+1,0\right)}^{\min \left(L_{a i}-1, D u\right)}}{\sqrt{\sum_{l=0}^{L_{a i}-1} P_{i}^{a}[l]}} \sqrt{\sum_{l=0}^{L_{a j}-1} P_{j}^{a}[l]} \\
& +\sum_{\substack{k=0 \\
k \neq\left(L_{a j}-1\right) / D}}^{\left(2 L_{a j}-2\right) / D} \sqrt{\sum_{l=0}^{L_{a i}-1} P_{i}^{a}[l]} \frac{\sum_{l=\max \left(D k-L_{a j}+1,0\right)}^{\min \left(L_{a j}-1, D k\right)} m_{a j}^{*}[l] m_{a j}\left[L_{a j}-1-D k+l\right]}{\sqrt{\sum_{l=0}^{L_{a j}-1} P_{j}^{a}[l]}} \\
& +\sqrt{\sum_{l=0}^{L_{a i}-1} P_{i}^{a}[l]} \sqrt{\sum_{l=0}^{L_{a j}-1} P_{j}^{a}[l]}
\end{aligned}
$$

\section{B. Passive user's case}

In this part, the expected SNR for a passive user (un-intended receiver) is compared to the one obtained for the active user. The received signal from the transmitter at the victim receiver may be expressed as:

$$
y_{p}[k]=\sum_{i=1}^{M} \sum_{l=0}^{\left(2 L_{i}-2\right) / D}\left(h_{p i} * g_{i}\right)[D l] x[k-l]+n_{p}[k],
$$

where $L_{i}=\left(L_{a i}+L_{p i}\right) / 2$. Since the SNR for a passive user depends on each CIR realization, the average $\mathrm{SNR}^{\mathrm{P}}$ is given by

$$
\begin{aligned}
E\left[S N R_{i}^{p}\right] & =\frac{E\left(P_{r}\right)}{\sigma_{p}^{2}} \\
& =\frac{P}{\gamma \sigma_{p}^{2}}\left(\sum_{u=0}^{(2 L-2) / D}\left[C_{i}^{\prime}(u)+D_{i}^{\prime}(u)\right]+A_{i}^{\prime \prime}+B_{i}^{\prime \prime}\right),
\end{aligned}
$$

where $A_{i}^{\prime \prime}, B_{i}^{\prime \prime}, C_{i}^{\prime}$ and $D_{i}^{\prime}$ are given in (26), (27), (28) and (29) respectively. Note that that the average $S N R^{p}$ depends on the power delay profile or the total energy of the active user.

Then,

$$
E\left(S N R_{M I S O}^{p}\right)=\sum_{i=1}^{M} S N R_{i}^{p}+\frac{P}{\sigma_{p}^{2}} \sum_{i=1}^{M} \sum_{\substack{j=1 \\ j \neq i}}^{M} B_{i j}^{\prime}
$$

with $L_{p i}$ and $L_{p j}$ are equal to the number of channel taps between the passive user and the considered small cell $i$ and $j$, and with $B_{i j}^{\prime}$ given by (31).

The choice of an active user affects then directly the EM pollution of the passive users. Hence, an efficient choice of the active user of interest has to be used as a candidate receiver. The goal will be, therefore, to minimize the sum of $E\left(S N R^{p}\right)$ on all the passive users.

\section{OptiMIZATION}

Consider a scenario with $K$ active users and $M$ passive users. A specific active user has to be chosen in order to minimize the electromagnetic waves on the majority of passive users. On the other hand, $E\left(S N R^{a}\right)$ is a discontinuous function and its optimization is not trivial. Out of the possible strategies, the following algorithm may be adopted

$$
\min _{i \in\{\mathcal{A}\}} \sum_{j=1}^{M} E\left[S N R_{j}^{p}\right]
$$

where $\mathcal{A}$ is the set of the active users and $i, j$ are the active and passive user's indexes, respectively.

\section{Simulation Results}

\section{A. Validation of the theoretical results}

In order to validate the theoretical results, Monte Carlo simulations have been carried out averaging over 100 channel realizations, taking the same power delay profile and the same number of taps for passive and active users. For completeness, different PDPs for active and passive users with different length of taps have also been considered. Let $L_{a}=L_{p}=16$, $M=4$ and all the channel taps distributed according to the same Gaussian distribution $N(0,1)$ (rectangle distribution). Additionally, we let $D \in[1,40]$. We note that, in order to show the relationship between the sampling rate and the average $\mathrm{SNR}$ at the receiver, the latter has been computed as 


$$
\begin{aligned}
& A_{i}^{\prime \prime}=\sum_{i=0}^{\left(2 L_{i}-2\right) / D} \sum_{\substack{j=0 \\
j \neq i}}^{\left(2 L_{i}-2\right) / D} \sum_{l=\max \left(i D-L_{a i}+1, j D-L_{a i}+1,0\right)}^{\min \left(L_{p i}-1, i D, j D\right)} P_{i}^{p}[l] m_{a i}^{*}\left[L_{a i}-1-i D+l\right] m_{a i}\left[L_{a i}-1-j D+l\right] \\
& B_{i}^{\prime \prime}=\sum_{i=0}^{\left(2 L_{i}-2\right) / D} \sum_{\substack{j=0 \\
j \neq i}}^{\left(2 L_{i}-2\right) / D} \sum_{l=\max \left(i D-L_{a i}+1,0\right)}^{\min \left(L_{p i}-1, i D\right)} \sum_{\substack{k=\max \left(j D-L_{a i}+1,0\right) \\
l \neq k}}^{\min \left(L_{p i}-1, j D\right)} m_{p i}[l] m_{p i}^{*}[k] m_{a i}\left[L_{a i}-1-j D+k\right] m_{a i}^{*}\left[L_{a i}-1-i D+l\right] \\
& C_{i}^{\prime}=\sum_{l=\max \left(D u-L_{a i}+1,0\right)}^{\min \left(L_{p i}-1, D u\right)} P_{i}^{p}[l] P_{i}^{a}\left[L_{i}-1-D u+l\right] \\
& D_{i}^{\prime}=\sum_{l=\max \left(D u-L_{a i}+1,0\right)}^{\min \left(L_{p i}-1, D u\right)} \sum_{\substack{k=\max \left(D u-L_{a i}+1,0\right) \\
k \neq l}}^{\min \left(L_{p i}-1, D u\right)} m_{p i}[l] m_{p i}^{*}[k] m_{a i}\left[L_{a i}-1-D u+k\right] m_{a i}^{*}\left[L_{a i}-1-D u+l\right]
\end{aligned}
$$

$$
B_{i j}^{\prime}=\frac{\sum_{u=0}^{\left(2 L_{i}-2\right) / D} \sum_{k=0}^{\left(2 L_{j}-2\right) / D} \sum_{l=\max \left(D u-L_{a i}+1,0\right)}^{\min \left(L_{p i}-1, D u\right)} \sum_{e=\max \left(D k-L_{a j}+1,0\right)}^{\min \left(L_{p j}-1, D k\right)} m_{p i}[l] m_{p j}^{*}[e] m_{a i}^{*}\left[L_{a i}-1-D u+l\right] m_{a j}\left[L_{a j}-1-D k+e\right]}{\sqrt{\sum_{l=0}^{L_{a i}-1} P_{i}^{a}[l]} \sqrt{\sum_{l=0}^{L_{a j}-1} P_{j}^{a}}}
$$

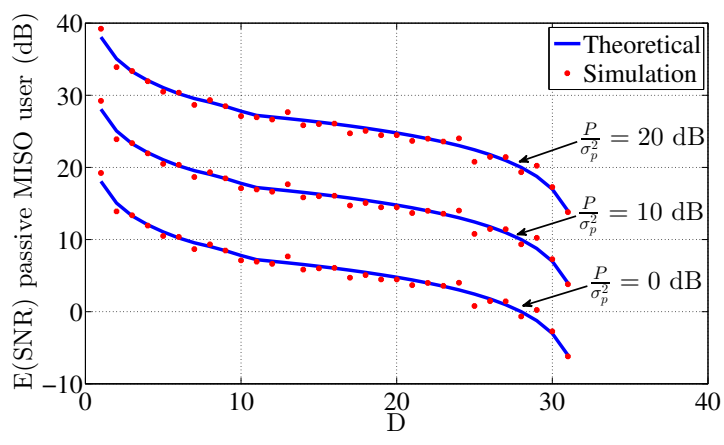

Figure 3. MISO passive user with rectangle distribution.

a function of the former. Fig. 3 shows the full match between the theoretical study and simulation in the passive user case with 3 plots corresponding to 3 different values of $\frac{P}{\sigma_{p}^{2}}$.

\section{B. Optimization}

In order to show the outcome of the optimization method described in the previous Section, let $\mathcal{A}=6$ and $M=8$. The Rayleigh fading channel's characteristics considered in the simulation are described in Table I, for all the passive users. By looking at Fig. 4 (Unoptimized) and Fig. 5 (Optimized), we can see that $\sum_{j=1}^{M} E\left[S N R_{j}^{p}\right]$ decreases when the number of active users increase if a good optimization process is applied (exploit the diversity effect brought by the higher number of users).
Table I

USERS’ DISTRIBUTION.

\begin{tabular}{|l|c|c|}
\hline Active User Number & Number of Taps & PDP Distribution \\
\hline User 1 & 50 & Exponential \\
User 2 & 100 & Random \\
User 3 & 120 & Random \\
User 4 & 150 & Rectangle \\
User 5 & 180 & Exponential \\
User 6 & 200 & Exponential \\
\hline \hline Passive User Number & Number of Taps & PDP Distribution \\
\hline User 1 & 100 & Exponential \\
User 2 & 80 & Exponential \\
User 3 & 110 & Exponential \\
User 4 & 90 & Exponential \\
User 5 & 120 & Exponential \\
User 6 & 130 & Exponential \\
User 7 & 150 & Exponential \\
User 8 & 40 & Exponential \\
\hline
\end{tabular}

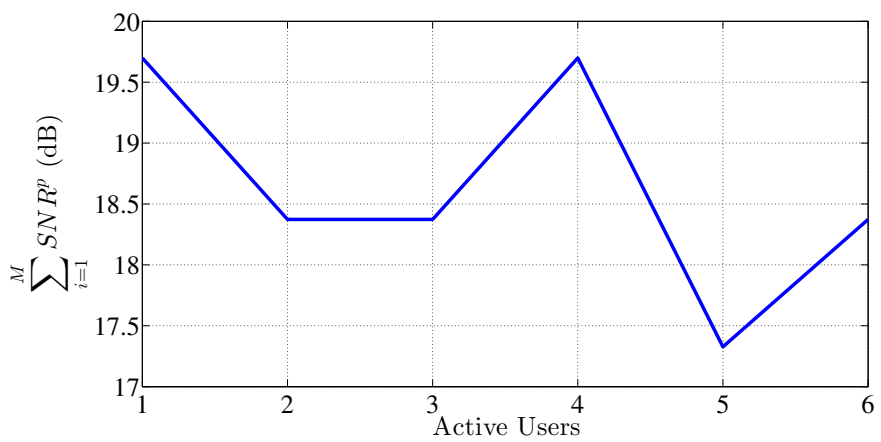

Figure 4. Sum of expected $S N R^{p}$ versus number of active usersUnoptimized. 


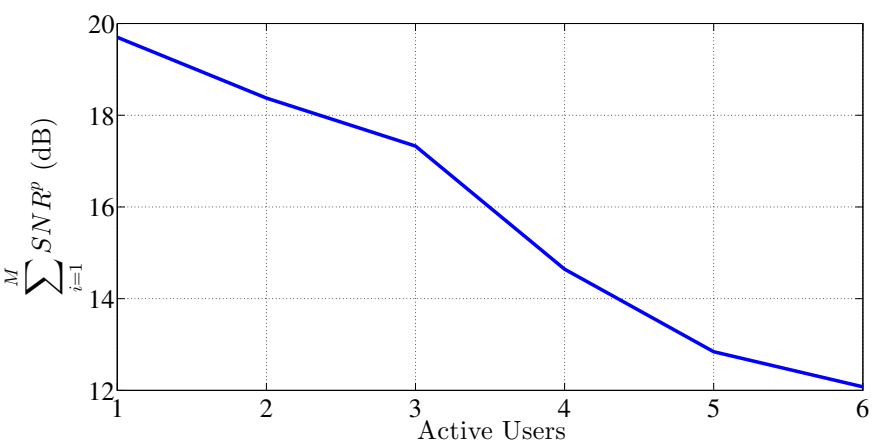

Figure 5. Sum of expected $S N R^{p}$ versus number of active users-Optimized.

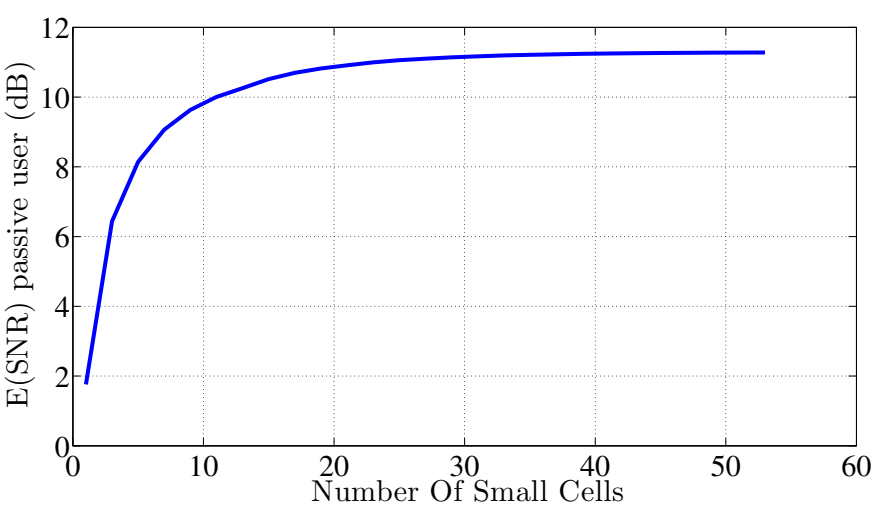

Figure 6. $\mathrm{E}(\mathrm{SNR})$ of the considered passive user.

\section{Deployment Limit}

In order to show the sufficient number of small cells needed for a working TR-base implementation, we consider 87 small cells as described in Fig. 1, letting the number of multi-path between the considered user and the small cell $i$ be $\frac{1}{\alpha^{i}}$, where $\alpha$ related to the path-loss coefficient factor, and the received power from each small cell is normalized by $\alpha^{3 i}$. Taking $\alpha=$ 0.9 the resulting threshold is 59 small cells (Fig. 6), distributed around the considered user. Note that $\mathrm{E}(\mathrm{SNR})$ saturates after a certain number of small cells. In fact, with farther smallcells there are more chances to have long channel impulse responses. After a certain threshold the power corresponding to far small cells become negligible and not affect the SNR experienced by passive user.

\section{CONCLUSiOnS}

In this paper, we proved that the SNR of active and passive users depends only on the power delay profile of channels between the base station and the considered users. Moreover, we saw how the SNR at the receiver is directly affected by the choice of the back-off factor $D$. Thus, the latter is an important parameter driving the choice of the best active user to serve. Our numerical findings show that TR can effectively decrease the EM pollution towards passive users. Accordingly, an appropriate optimization has to be performed to achieve this goal and increase the overall system performance. As TR works only with high number of multi-paths, we solve this restriction by deploying a network of small cells. Finally, TR could be used in a network of small cells and may be one of the original technique to focus the most amount of energy to the considered user techniques (active user) and decrease the energy toward all the other users (passive+active).

\section{REFERENCES}

[1] J. Hoydis, M. Kobayashi, and M. Debbah, "Green small-cell networks," IEEE Vehicular Technology Magazine, vol. 6, pp. 37-43, March 2011 2011.

[2] M. Seifeldin, A. Saeed, A. Kosba, A. El-keyi, and M. Youssef, "Nuzzer: A large-scale device-free passive localization system for wireless environments," Mobile Computing, IEEE Transactions on, vol. PP, no. 99, p. 1, 2012.

[3] M. Youssef, M. Mah, and A. Agrawala, "Challenges: device-free passive localization for wireless environments," in Proceedings of the 13th annual ACM international conference on Mobile computing and networking, ser. MobiCom 07, 2007, pp. 222-229.

[4] N. Patwari and J. Wilson, "Rf sensor networks for device-free localization: Measurements, models, and algorithms," Proceedings of the IEEE, vol. 98, no. 11, pp. $1961-1973$, nov. 2010.

[5] B. Wang, Y. Wu, F. Han, Y.-H. Yang, and K. Liu, "Green wireless communications: A time-reversal paradigm," Selected Areas in Communications, IEEE Journal on, vol. 29, no. 8, pp. 1698 -1710, september 2011.

[6] G. Lerosey, J. de Rosny, A. Tourin, A. Derode, G. Montaldo, and M. Fink, "Time reversal of electromagnetic waves," Phys. Rev. Lett., vol. 92, p. 193904, May 2004.

[7] F. Monsef and A. Cozza, "Analysis of time-reversal-based propagation for spatial focusing and multiplexing," in Antennas and Propagation in Wireless Communications (APWC), 2011 IEEE-APS Topical Conference on, sept. 2011, pp. $827-830$.

[8] P. Roux, J. de Rosny, M. Tanter, and M. Fink, "The aharonov-bohm effect revisited by an acoustic time-reversal mirror," Phys. Rev. Lett., vol. 79, pp. 3170-3173, Oct 1997.

[9] M. Fink, "Time reversal of ultrasonic fields. i. basic principles," $U l$ trasonics, Ferroelectrics and Frequency Control, IEEE Transactions on, vol. 39, no. 5, pp. 555 -566, sept. 1992.

[10] J. Hansen, D. S. Baum, and A. J. Paulraj, "Equipment for time reversal in wireless communications," in Special session on MIMO prototyping, Swiss Federal Institute, 2004.

[11] F. Monsef, A. Cozza, and L. Abboud, "Effectiveness of time-reversal technique for uwb wireless communications in standard indoor environments," in ICECom, 2010 Conference Proceedings, sept. 2010, pp. 1 $-4$.

[12] P. Kyritsi, G. Papanicolaou, P. Eggers, and A. Oprea, "Miso time reversal and delay-spread compression for fwa channels at $5 \mathrm{ghz}$," Antennas and Wireless Propagation Letters, IEEE, vol. 3, no. 1, pp. 96 -99, dec. 2004.

[13] M. Debbah, "Tutorial on mimo technologies," Alcatel-Lucent Radio on Flexible Radio, Supélec, [Online] Available: http://flexibleradio.com/teaching/course-materials, 2008.

[14] M. Emami, M. Vu, J. Hansen, A. J. Paulraj, and G. Papanicolaou, "Matched filtering with rate back-off for low complexity communications in very large delay spread channels," in Proc.38th Asilomar Conference Signals, Syst. Comput., vol. 1,pp.218-222, Nov. 2004.

[15] B. Mawlawi, "Master report," Supélec, Tech. Rep., 2012. 